\title{
Occlusion ambiguities in motion
}

\author{
Davi Geiger ${ }^{1}$ and Kostas I. Diamantaras ${ }^{2}$ \\ 1 Courant Institute, NYU, 251 Mercer street, New York NY 10012, USA \\ 2 Siemens Corporate Research, 755 College Rd. East, New Jersey NJ 08540
}

\begin{abstract}
We address the problem of optical flow reconstruction and in particular the problem of resolving ambiguities near edges. They occur due to (i) the aperture problem and (ii) the occlusion problem, where pixels on both sides of an intensity edge are assigned the same velocity estimates (and confidence). However, these measurements are correct for just one side of the edge (the non occluded one). We note that the confidence measures are large at intensity edges and larger at the convex sides of the edges, i.e. inside corners, than at the concave side. We resolve the ambiguities through local interactions via coupled Markov random fields (MRF). The result is the detection of motion for regions of images with large global convexity.
\end{abstract}

\section{Introduction}

In this paper we discuss the problem of figure ground separation, via optical flow, for homogeneous images (textured images just provide more information for the disambiguation of figure-ground). We address the problem of optical flow reconstruction and in particular the problem of resolving ambiguities near intensity edges. We concentrate on a two frames problem, where all the motion ambiguities we discuss can be disambiguiated by the human visual system.

Optical flow is a 2D (two dimensional) field defined as to capture the projection of the 3D (three dimensional) motion field into the view plane (retina). The Horn and Schunk[9] formulation of the problem is to impose (i) the brightness constraint $\frac{d E(x, y, t)}{d t}=0$, where $E$ is the intensity image, and (ii) the smoothness of the velocity field.

We utilize the following optical flow computation improvements, (i) the introduction of the confidence measure (Nagel and Enkelman [11], Anandan [1]) and (ii) the application of smoothness while preserving discontinuities (Geman and Geman [6], Blake and Zisserman [2], Mumford and Shah [10]). It is clear that as an object moves with respect to a background not only optical flow discontinuities occur, but also occlusions occur (and revelations). In stereo, occlusions are related to discontinuities (e.g. Geiger et. al 1992 [5]), and for motion a similar relation must exist. We study ambiguities ocuring at motion discontinuities and occlusions. 


\section{Motion ambiguities}

Figure 1 shows two synthetic problems involving a translation and a rotation of simple objects in front of stationary backgrounds.

Consider the case of the square translation (see figure 1a.). Humans perceive the square translating, although block-matching (and any other matching technique) gives translation on both sides of the square edges. Moreover, there are other interpretations of the scene, such as the square belonging to the stationary background and the outside being a translating foreground with a square hole. The examples are synthetic, but emphasize the ambiguities. Real images may have more texture, thus many times helping resolve these ambiguities, but not everywhere.

\section{A Markov random field model}

We describe a model capable of solving these ambiguities. It is based on coupled Markov random fields and thus, based on local processes. Our main contribution is to introduce the idea of uncertainty on the estimates and on the confidence measures. We propose a Markov field that allows the estimates of each pixel to be chosen among a large neighborhood, thus each pixel estimate can be neglected.

\subsection{Block Matching}

Block matching is the process of correlating a block region of one image, say of size $\left(2 \omega_{M}+1\right) \times\left(2 \omega_{M}+1\right)$, with a block region of the other image. Block-matching yields a set of matching errors $d_{i j}^{m n}$, where $(i, j)$ is a pixel in the image and $v=[m, n]$ is a displacement vector in a search window of size $\left(2 \omega_{S}+1\right) \times\left(2 \omega_{S}+1\right)$ around the pixel. We define the velocity measurements $g_{i j}$ and the covariance matrix $C_{i j}$ as the mean and variance of the vector $v=[m, n]$ averaged according to the distribution $e^{-k d_{i j}^{m n}}$ :

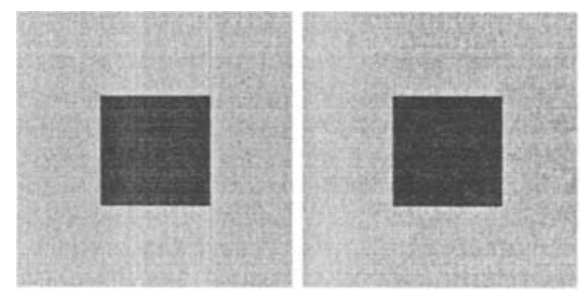

(a)

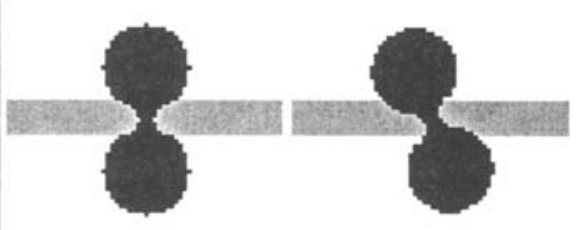

(b)

Fig. 1. Two image sequences of $128 \times 128$. (a) Square translation of 3 pixels; (b) "Eight" rotation of $10^{\circ}$. Note that the "eight" has concave and convex regions. 


$$
g_{i j}=\frac{\sum_{m, n} e^{-k d_{i j}^{m n} v}}{\sum_{m, n} e^{-k d_{i j}^{m n}}} \quad C_{i j}=\frac{\sum_{m, n} e^{-k d_{i j}^{m n}\left(v-g_{i j}\right)\left(v-g_{i j}\right)^{T}}}{\sum_{m, n} e^{-k d_{i j}^{m n}}}
$$

Figure 2 shows the block matching data $g_{i j}$ for the two problems discussed above and figure 3 shows the correspondent confidence measurse (inverse of the covariance matrix as defined below).

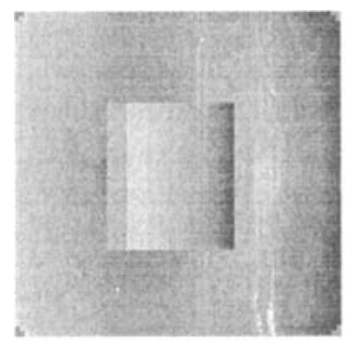

(a)

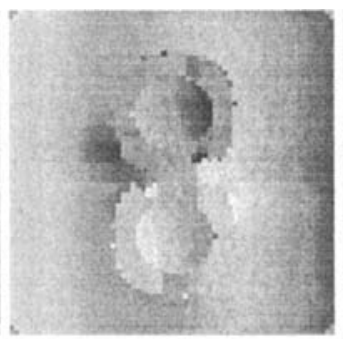

(b)

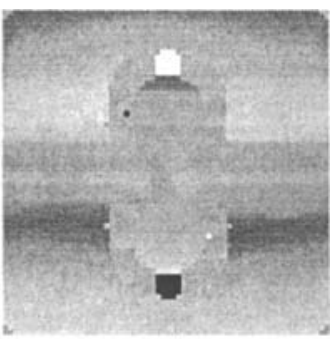

(c)

Fig. 2. Block matching data $g_{i j}$. Both sides of the edges have the same data (and same confidence). White represents motion to the right ( $\mathrm{x}$-direction) or up (y-direction). Black is the complement. (a) The $x$-component of the data for the square translation. (b) The $x$-component of the data for the rotation and (c) the y-component of the data.

\subsection{The aperture problem and confidence}

The aperture problem [8] occurs where there is a low confidence on the measurements (data) in the direction along an edge; In particular we follow the approach by [1], through the use of the confidence matrix defined as

$$
R_{i j}=\epsilon\left(C_{i j}+\epsilon\right)^{-1}
$$

where $\epsilon$ is small to guarantee inverse. The confidence measures at straight edges is high perpendincular to the edges and low (zero) along the edges. However, at corners, the confidence is high on both directions thus through smoothness this result can be propagated through the other parts of the image, then resolving the aperture problem.

\subsection{The localization problem and a binary decision field}

The localization problem arises due to the local symmetry at intensity edges, where both sides of an edge give the same correspondences. These cases occur 
when occluded regions are homogeneous and so, block matching, pixel matching or any matching technique can not distinguish which side of the edge is being occluded or is occluding. Even if one considers edge based methods, the same problem arises in the reconstruction stage, where the edge velocities have to be propagated to the rest of the image.

We note however, a bias towards inside of corner regions from the confidence measures. In a corner, despite both sides getting roughly the same velocity estimate and high confidence measures, the inside pixel always get a larger confidence (see figure $3 \mathrm{c}, \mathrm{d}$ ). This bias is due to having more pixels outside the edge of a closed contour than outside, and occurs at the convex regions (e.g. a corner). It can be corrected (e.g. [7]), but we want to explore this bias. Thus, in general, the convex regions will have a stronger confidence measure than outside them. Note that at concavities in the "eight" rotation image, the confidence will be higher outside the "eight" and correct at convex regions. Thus, a global optimization will be required to decide which confidences to "pick up".

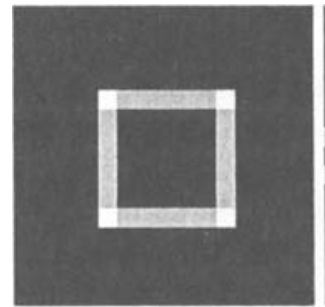

(a)

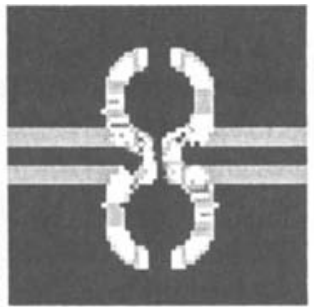

(b)

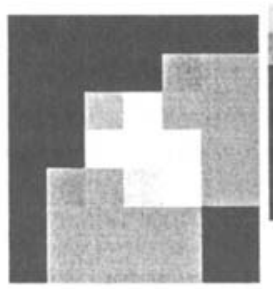

(c)

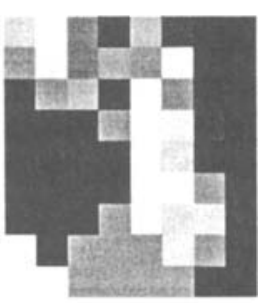

(d)

Fig. 3. The confidence $R$ extracted from the block matching data $g_{i j}$. The display is the sum of both eigenvalues, i.e. the trace of $R$. Both sides of the edges have the same confidence. White represents high confidence. (a) For the square translation. (b) For the rotation. (c) a close up on the top left corner. (d) a close up on the concavitiy of the eight shape.

Our approach to resolve this ambiguity is to allow for the motion estimate at pixel $(i, j)$ to select data from a neighborhood $N_{i j}$, and its goal is to maximize the total estimates (taking into account the confidence measures). More precisely, let $f_{i j}$ be the vector motion field at pixel $(i, j)$. We introduce a binary field $\alpha_{i j}^{m n}$ that indicates which data $g_{i+m, j+n}$ in a neighborhood $N_{i j}$ of $(i, j)$ should correspond to a motion estimate $f_{i j}$. The size of $N_{i j}$ is given by $\omega_{M}+1$ to overcome the localization uncertainty. For a given lattice point $(i, j)$ the boolean parameters $\alpha_{i j}^{m n}$ should be mutually exclusive, i.e. only one of them, $\alpha_{i j}^{m n^{*} n^{*}}$, should be equal to 1 indicating that $f_{i j}$ should correspond to $g_{i+m^{*}, j+n^{*}}$, while the rest $\alpha_{i j}^{m n}$, $m \neq m^{*}, n \neq n^{*}$, should be zero (or $\sum_{m^{*} n^{*} \in N_{i j}} \alpha_{i j}^{m^{*} n^{*}}=1$ ). The conditional probability reflects both an uncertainty due to noise and an uncertainty due to 
spatial localization of the data

$$
P(R, g \mid f, \alpha)=\frac{1}{C_{2}} \exp \left\{-\sum_{i j} \sum_{m n \in N_{i j}} \alpha_{i j}^{m n}\left\|R_{i+m, j+n}\left(f_{i j}-g_{i+m, j+n}\right)\right\|^{2}\right\}
$$

where $\|h\|^{2}=h_{x}^{2}+h_{y}^{2}$ for $h=\left[h_{x}, h_{y}\right]$.

\subsection{Piecewise smooth prior and posterior distribution}

The prior probability of the motion field $f_{i j}$ is a piecewise smoothness condition,

$$
P(f, \alpha, h, v)=\frac{1}{C_{1}} e^{-\left(\sum_{i j} \mu\left(1-h_{i j}\right)\left\|f_{i j}-f_{i-1, j}\right\|^{2}+\mu\left(1-v_{i j}\right)\left\|f_{i j}-f_{i, j-1}\right\|^{2}+\gamma_{i j}\left(h_{i j}+v_{i j}\right)\right)},
$$

where $h_{i j}=0\left(v_{i j}=0\right)$ if there is no motion discontinuity separating pixels $(i, j)$, $(i-1, j)((i, j),(i, j-1))$, otherwise $h_{i j}=1\left(v_{i j}=1\right)$. The parameter $\mu$ has to be estimated. We have considered that the cost to create motion discontinuities should be lowered at intensity edges (see Poggio et al. [12]), i.e $\gamma_{i j}=\gamma\left(1-\delta e_{i j}\right.$ ), where $e_{i j}$ is the intensity edge and $0 \leq \delta \leq 1$ and $\gamma$ have to be estimated.

The posterior distribution is given by Bayes' law

$$
P(f, \alpha, h, v \mid g, R)=\frac{1}{P(g, R)} P(g, R \mid f, \alpha) P(f, \alpha, h, v)
$$

To obtain a close MAP estimate we use statistical physics techniques described in [3].

\section{Results}

Figure 4 depicts the results of the experiments. The system chooses a natural interpretation, namely the object (e.g. the square in the first example or the eight-shaped region in the second example) moving and the background being stationary. We have also applied to real images and the results support the method. A main drawback is the computational time.

Acknowledgements The first author would like to thank the Isaac Newton Institute for Mathematics Sciences for providing an outstanding environment, where part of this research was conducted (and special thanks for the criticism of A. Blake and J. Mayhew, where they argue that higher level approaches may be more appropriate). Also, it was noted by a referee that Thompson et al, Pami, July 85; Heitz et. al, ICPR'90; and Depommier et. al, ICASSP'92 have already addressed the occlusion ambiguities, perhaps in a different manner then we present here. 


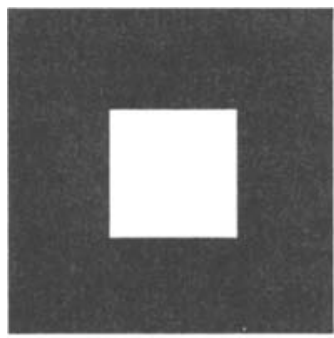

(a)

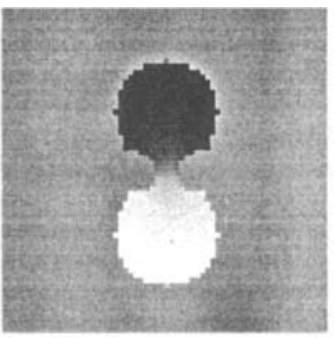

(b)

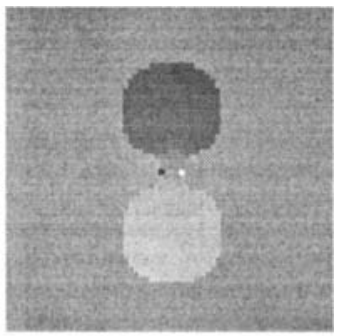

(c)

Fig. 4. The final motion estimation, after 20000 iterations, resolved the ambiguities with a natural interpretation of the scene. $\mu=10, \delta=1, \gamma=100$. (a) square translation (b) $\mathrm{x}$ component of the motion rotation (c) $\mathrm{y}$ component of the motion rotation

\section{References}

1. P. Anandan, "Measuring Visual Motion from Image Sequences", PhD thesis, COINS Dept., Univ. Massachusetts, Amherst, 1987.

2. A. Blake and A. Zisserman, "Visual Reconstruction", Cambridge, Mass, MIT press, 1987.

3. K.I. Diamantaras and D. Geiger, "Resolving motion ambiguities", Proceedings of Neural Information Processing Systems, vol 6, 1994.

4. D. Geiger and F. Girosi, "Parallel and Deterministic Algorithms for MRFs: Surface Reconstruction and Integration", IEEE PAMI: 13(5), May 1991.

5. D. Geiger and B. Ladendorf and A. Yuille, "Binocular stereo with occlusion", Computer Vision- ECCV92, ed. G. Sandini, Springer-Verlag, 588, pp 423-433, May 1992.

6. S. Geman and D. Geman, "Stochastic Relaxation, Gibbs Distributions, and the Bayesian Restoration of Images", IEEE PAMI 6, pp. 721-741, 1984.

7. Z. Geyzel and M. Werman "Conic Section Fitting", Tech. Rep. Hebrew University, 1994.

8. E. C. Hildreth, "The measurement of visual motion", MIT press, 1983.

9. B.K.P. Horn and B.G. Schunk, "Determining optical flow", Artificial Intelligence, vol 17, pp. 185-203, August 1981.

10. D. Mumford and J. Shah, "Boundary detection by minimizing functionals, I", Proc. IEEE Conf. on Computer Vision \& Pattern Recognition, San Francisco, CA, 1985.

11. H.-H. Nagel and W. Enkelmann, "An Investigation of Smoothness Constraints for the Estimation of Displacement Vector Fields from Image Sequences", IEEE PAMI: 8, 1986.

12. T. Poggio and E. B. Gamble and J. J. Little, "Parallel Integration of Vision Module”, Science, vol 242, pp. 436-440, 1988.

13. S. Ullman, "The Interpretation of Visual Motion", Cambridge, Mass, MIT press, 1979 . 